\title{
Disruption und Innovationsforschung
}

\author{
Ingo Schulz-Schaeffer
}

Die Innovationsforschung entstand im 20. Jh. wesentlich in Auseinandersetzung mit disruptiven Neuerungen, während kontinuierliche Weiterentwicklungen zunächst als weniger interessant galten. Sie kamen stärker in den Blick, als empirische Forschungen zeigten, dass diese dann als inkrementelle Innovationen bezeichneten kontinuierlichen Weitentwicklungen von wesentlicher Bedeutung bei der Stabilisierung und Durchsetzung disruptiver Neuerungen sind. Zunehmend entwickelte sich in der Innovationsforschung ein komplexeres Bild der Verwobenheit kontinuierlicher und diskontinuierlicher Neuerungen. Einerseits wurde deutlich, dass es Konstellationen gibt, in denen diskontinuierliche Entwicklungen in kontinuierliche Entwicklungen integriert werden; andererseits wurden Konstellationen sichtbar, in denen die Disruption diskontinuierlicher Entwicklungen als Effekt kontinuierlicher Entwicklungen noch gesteigert wird. In meinem Vortrag werde ich diese Entwicklungen der Innovationsforschung in Grundzügen nachzeichnen.

\section{Diskontinuität und Handlungsunsicherheit}

Aus heutiger Sicht bildet Joseph Schumpeters Theorie der wirtschaftlichen Entwicklung von 1911 den Grundstein der Theorieentwicklung der Innovationsforschung im 20. Jh. Schumpeter verwendet den Begriff der Disruption noch nicht - dieser Begriff ist erst seit gut zwei Jahrzehnten Bestandteil des Vokabulars der Innovationsforschung. Von der Sache her geht es Schumpeter aber in zentraler Hinsicht um disruptive Entwicklungen.

Schumpeters Interesse gilt dem, was er als diskontinuierliche Entwicklungen im wirtschaftlichen Leben bezeichnet. Eines seiner Beispiele für eine solche diskontinuierliche Entwicklung ist die Ablösung der Postkutsche durch die Eisenbahn. Schumpeter interessiert sich für diskontinuierliche Entwicklungen im Wirtschaftsleben, weil sie ein wirtschaftswissenschaftliches Erklärungsproblem darstellen: Im Gegensatz zu kontinuierlichen wirtschaftlichen Entwicklungen lassen sie sich nicht im Rahmen der seinerzeit vorherrschenden ökonomischen Gleichgewichtstheorien erfas- 
sen und verstehen. Diese Theorien, so Schumpeter, erfassen das Wirtschaftsleben unter dem „Gesichtspunkt einer Tendenz der Volkswirtschaft nach einem Gleichgewichtszustand, welche Tendenz uns die Mittel gibt, die Preise und Mengen der Güter zu bestimmen, und sich als Anpassung an jeweils vorhandene Daten darstellt. "1 Dies heißt, „die einzelnen Vorgänge in der Volkswirtschaft als Teilerscheinungen der Tendenz nach einem Gleichgewichtszustand begreifen. “2

Aus dieser Perspektive ist wirtschaftliches Handeln ein Prozess der kontinuierlichen Anpassung an einen gegebenen ideellen Gleichgewichtszustand. Wirtschaftliches Handeln findet demnach immer in demselben Rahmen statt, was den Wirtschaftssubjekten die Mittel an die Hand gibt, ihr Handeln in Kategorien von Preisen und Gütermengen ökonomisch rational zu kalkulieren, und der Wirtschaftswissenschaft die Mittel an die Hand gibt, die resultierenden ökonomischen Prozesse zu verstehen und zu erklären.

„Allein diese Mittel versagen“, so Schumpeter, „wo das wirtschaftliche Leben selbst seine eigenen Daten ruckweise ändert. Bau einer Eisenbahn kann [...] als Beispiel dienen. Kontinuierliche Veränderungen, die mit der Zeit in steter Anpassung mittels zahlloser kleiner Schritte aus einem kleinen Detailgeschäft ein großes, z. B. ein Warenhaus, machen können, fallen unter die statische Betrachtung. Aber nicht [...] fundamentale Veränderungen in der Sphäre der Produktion im weitesten Sinn: Da kann sie [d.h. die gleichgewichtstheoretische Betrachtung, Anm. ISS] nicht nur die Folgen mit ihren auf die Infinitesimalmethode eingestellten Mitteln nicht präzise voraussagen, sondern sie kann weder das Zustandekommen solcher produktiven Revolutionen erklären, noch die Erscheinungen die dabei auftreten, - sondern nur, wenn sie vorgefallen sind, den neuen Gleichgewichtszustand untersuchen. “3

Diskontinuierliche Entwicklungen sind dadurch gekennzeichnet, dass sie "den Rahmen, die gewohnte Bahn selbst verändern." 4 Sie entziehen sich deshalb der gleichgewichtstheoretischen Betrachtung. Schumpeter charakterisiert diese diskontinuierlichen Veränderungen im Wirtschaftsleben allgemein als „Durchsetzung neuer Kombinationen von Produktionsmitteln“

1 Schumpeter, Theorie der wirtschaftlichen Entwicklung. Eine Untersuchung über Unternehmergewinn, Kapital, Kredit, Zins und den Konjunkturzyklus, 4. Auflage, 1934 [1911], S. 94.

2 Schumpeter, ebd., S. 94.

3 Schumpeter, ebd., S. 95.

4 Schumpeter, ebd., S. 93. 
und fasst darunter neue Produkte, neue Produktionsverfahren, neue Organisationsformen und andere Veränderungen, die die Wirkung haben, die Spielregeln für die von ihnen betroffenen Wirtschaftssubjekte zu verändern. ${ }^{5}$

Mit der Charakterisierung diskontinuierlicher Entwicklungen als Veränderungen, die den Rahmen des wirtschaftlichen Handelns selbst betreffen, beschreibt Schumpeter sie als Phänomene, die für Wirtschaftssubjekte zugleich sehr erstrebenswert und sehr nachteilig sind bzw. sein können. Hochgradig erstrebenswert sind sie in einer Konkurrenzwirtschaft, weil sie denjenigen Wirtschaftssubjekten Wettbewerbsvorteile verschaffen können, die die neuen Rahmenbedingungen exklusiv für sich nutzbar machen können oder schneller für sich nutzbar machen können als andere. Zugleich entwerten die wirtschaftlich erfolgreichen neuen Produkte und Prozesse die alten, ein Prozess, den Schumpeter später als schöpferische Zerstörung („,creative destruction“) bezeichnet hat. ${ }^{6}$ Für die Besitzer der alten Produktionsmittel sind sie deshalb zunächst nachteilig.

Die von Schumpeter in den Blick genommenen diskontinuierlichen Entwicklungen im Wirtschaftsleben tragen gegenüber den kontinuierlichen Entwicklungen noch einen zweiten Nachteil in sich, von dem deren spätere Profiteure genauso betroffen sind wie die späteren Modernisierungsverlierer: Sie lassen sich nicht rational kalkulieren. In den Worten von Schumpeter:

„Während im gewohnten Kreislauf jedes Wirtschaftssubjekt, seines Rodens sicher und getragen von dem auf diesen Kreislauf eingestellten Verhalten aller andern Wirtschaftssubjekte, mit denen es zu tun hat und die ihrerseits wieder das gewohnte Verhalten von ihm erwarten, prompt und rationell handeln kann, so kann es das nicht ohne weiteres, wenn es vor einer ungewohnten Aufgabe steht. Während in gewohnten Bahnen dem normalen Wirtschaftssubjekt sein eigenes Licht und seine Erfahrung genügt, so bedarf es Neuem gegenüber einer Führung. Während es mit dem Strom schwimmt im allseits wohlbekannten Kreislauf, schwimmt es gegen den Strom, wenn es dessen Bahn verändern will. Was dort Stütze war, wird hier Hindernis. Was vertrautes Datum war, zu einer Unbekannten. Wo die Grenze der Routine aufhört, können deshalb viele Leute nicht weiter und der Rest kann es nur in sehr verschiedenem Maß.“7

5 Vgl. Schumpeter, Theorie der wirtschaftlichen Entwicklung, S. $100 \mathrm{f}$.

6 Vgl. Schumpeter, Capitalism, Socialism, and Democracy, 1942, S. 83.

7 Schumpeter, Theorie der wirtschaftlichen Entwicklung, S. $117 \mathrm{f}$. 
In seiner 1911 erschienenen Theorie der wirtschaftlichen Entwicklung, in der Schumpeter diese Überlegungen entwickelt, verwendet er den Begriff der Innovation noch nicht. In seinem 1939 veröffentlichten Buch Business Cycles $^{8}$ avanciert der Begriff der Innovation dagegen zu einem Zentralbegriff. Er dient dort zur Bezeichnung des Sachverhaltes, der bereits in der Theorie der wirtschaftlichen Entwicklung im Mittelpunkt steht: Innovation ist diskontinuierliche Veränderung qua Durchsetzung neuer Kombinationen von Produktionsfaktoren. ${ }^{9}$

Mit seiner Innovationstheorie nimmt Schumpeter eine Fokussierung des Gegenstandsbereichs vor, die die nachfolgende Innovationsforschung für lange Zeit prägen sollte: die Fokussierung auf Innovationen als fundamentale Neuerungen im Gegensatz zu kleinschrittigen Veränderungen. Und er wirft eine Forschungsfrage auf, die die nachfolgende Innovationsforschung für lange Zeit beschäftigen wird: Die Frage danach, was innovierende Akteure und Dritte, die von Innovationen in der einen oder anderen Weise betroffen sind, tun, tun können oder tun sollten, um mit der hochgradigen Handlungsunsicherheit umzugehen, die mit Innovationsprozessen verbunden ist.

\section{Handlungsunsicherheit und Heuristiken}

Der Umstand, dass Innovation grundlegend mit Unsicherheit verbunden ist, ist zentral für die Innovationstheorie, die Richard R. Nelson und Sidney G. Winter in einem wegweisenden Artikel aus dem Jahre 1977 mit dem Titel In search of a useful theory of innovation entwickeln. Ihr innovationstheoretischer Ansatz wird später eine ganze ökonomische Denkrichtung begründen, die Evolutionsökonomik („evolutionary economics“). Ganz im Sinne von Schumpeter argumentieren Nelson und Winter, dass es wegen der inhärenten Unsicherheit von Innovationsprozessen keine Produktionsfunktion für Innovativität geben kann, es also nicht möglich ist, bestimmte benennbare Faktorenkombinationen für den Erfolg oder Misserfolg von Innovationen zu identifizieren. Stattdessen betonen sie: „Wegen der bestehenden Unsicherheit werden unterschiedliche Menschen und verschiedene Organisationen stets unterschiedlicher Auffassung da-

8 Schumpeter, Business Cycles. A Theoretical, Historical and Statistical Analysis of the Capitalist Process, 1939.

9 Schumpeter, Konjunkturzyklen. Eine theoretische, historische und statistische Analyse des kapitalistischen Prozesses, Bd. 1, 1961 [1939], S. 95. 
rüber sein, wo sie ihre Forschungs- und Entwicklungseinsätze platzieren und wann sie ihre Einsätze tätigen sollen. Einige werden sich als richtig und andere als falsch herausstellen." 10

Mit Schumpeter sind sich Nelson und Winter auch einig, dass Innovationsprozesse wegen dieser inhärenten Unsicherheit aus dem Rahmen gleichgewichtstheoretischer Analysen fallen. Sie schreiben: „[W]ir behandeln jede Innovation sowohl vor als auch nach ihrer Einführung in die Wirtschaft als mit erheblicher Unsicherheit behaftet und sehen daher den Innovationsprozess als ein anhaltendes Ungleichgewicht an. Zu jeder Zeit gibt es eine Koexistenz von Ideen, die sich zu erfolgreichen Innovationen entwickeln werden, und solchen, bei denen dies nicht der Fall sein wird, und zu jeder Zeit sind zugleich profitable und falsch eingeschätzte oder veraltete Technologien in Gebrauch. Im Laufe der Zeit unterliegt das jeweils bestehende Set an Technologien einer Selektion, aber es werden ständig neue eingeführt, um die Bewegung in Richtung Gleichgewicht zu stören." 11

Im Gegensatz zu Schumpeter können Nelson und Winter in ihren Überlegungen auf Befunde aus gut zwei Jahrzehnten empirischer Innovationsforschung zurückgreifen, die in den USA etwa ab Anfang der 1950er Jahren als Erforschung des technologischen Wandels Fahrt aufgenommen hatte. ${ }^{12}$ Auf dieser Grundlage entwickeln die Autoren die folgenden Überlegungen zu den Bewältigungsstrategien von Unternehmen im Umgang mit der inhärenten Unsicherheit von Innovationsprozessen, die ein neues Licht auf das Verhältnis von kontinuierlichem und diskontinuierlichem Wandel werfen:

In Anbetracht der Unmöglichkeit, über Innovationsprozesse auf der Grundlage rationaler Kalkulation entscheiden zu können, weichen Unternehmen, so Nelson und Winter, auf Heuristiken aus. Sie entscheiden mit Hilfe heuristischer Suchprozesse, welche Forschungs- und Entwicklungsprojekte („F\&E-Projekte“) sie auswählen und durchführen. Auch die Entscheidungen innerhalb ihrer F\&E-Projekte beruht wesentlich auf Heuristiken. Unter einem heuristischen Suchprozess verstehen die Autoren „eine Aktivität, die ein Ziel hat und über eine Reihe von Verfahren verfügt, vielversprechende Wege zu identifizieren, zu durchleuchten und anzusteuern, um zu diesem Ziel oder in dessen Nähe zu gelangen. Die Verfahren kön-

10 Nelson/Winter, In Search of a Useful Theory of Innovation, in: Research Policy 6 (1977), S. 47, eigene Übersetzung.

11 Nelson/Winter, ebd., S. 48, eigene Übersetzung.

12 Godin, "Innovation Studies": The Invention of a Specialty (Part I), Project on the Intellectual History of Innovation Working Paper No. 7, 2010. 
nen durch den Einsatz von annähernder Ziele bestimmt sein, durch Fokussierung auf bestimmte Hinweise und Anhaltspunkte oder auch durch Faustregeln verschiedener Art. "13 Eine häufig verwendete Faustregel ist es beispielsweise, jährlich einen festgelegten Prozentsatz des Umsatzes in F\&E zu investieren.

Zusammengenommen bilden diese Heuristiken die F\&E-Strategie des Unternehmens. Häufig enthalten die F\&E-Strategien eine Beschränkung „auf eine oder wenige Klassen von F\&E-Projekten [...], die jeweils eine gewisse Ähnlichkeit in Bezug auf allgemein definierte Ziele, Verfahren zur Erreichung dieser Ziele und die erforderlichen F\&E-Ressourcen aufweisen. "14 In diesem Sinne fokussieren innovierende Unternehmen im Bereich der Landwirtschaft dann beispielsweise entweder auf Innovationen im Bereich landwirtschaftlicher Maschinen oder im Bereich des Saatgutes. Weil innerhalb einer solchen Klasse von F\&E-Projekten die eingesetzten Verfahren und die erforderlichen Ressourcen ähnlich sind, steht die F\&EStrategie in engem Zusammenhang mit den korrespondierenden Fähigkeiten, Ausstattungen und Organisationsstrukturen des betreffenden Unternehmens. ${ }^{15}$

Alle diese Heuristiken haben das Ziel, Entscheidungsunsicherheit zu reduzieren. Wie hier bereits deutlich wird, haben sie zudem die in diesem Zusammenhang besonders interessierende Eigenschaft, Elemente der Kontinuität in das grundsätzlich diskontinuierliche Geschehen des Innovierens hineinzubringen. Für diese Transformation von Diskontinuität in Kontinuität ist eine weitere Gruppe von Heuristiken von zentraler Bedeutung, die Nelson und Winter mit Hilfe der Konzepte der natürlichen Trajektorie und des technologischen Regimes beschreiben. Von besonderer Bedeutung sind sie vorallem deshalb, weil es dabei um Heuristiken geht, die nicht allein auf der Ebene einzelner Unternehmen, sondern auf der Ebene ganzer Branchen oder organisationaler Felder wirksam sind.

Mit dem Begriff der natürlichen Trajektorie („natural trajectory“) erfassen Nelson und Winter das Phänomen, dass existierende technische $\mathrm{Zu}$ sammenhänge vielfach gleichsam aus sich heraus auf machbare und vielversprechende Innovationsprojekte verweisen. Entsprechende Signale zeigen sich etwa in Form von Schwachstellen oder Engpässen, die es zu beheben oder aufzulösen lohnt. Diese Art von Signalen hat Thomas P. Hughes

13 Nelson/Winter, In Search of a Useful Theory of Innovation, S. 52f., eigene Übersetzung.

14 Nelson/Winter, ebd., S. 55, eigene Übersetzung.

15 Vgl. Nelson/Winter, ebd., S. 56. 
in seinen Überlegungen zur Evolution technischer Systeme sehr anschaulich mit der Metapher der reverse salients beschrieben. Ein reverse salient ist eine Einbuchtung in einer geometrischen Struktur, wie etwa die zurückhängende Frontlinie einer Wetterfront oder einer Kriegsfront. Bezogen auf technische Systeme sind reverse salients, so Hughes, „Komponenten des System, die in Rückstand geraten sind und mit den anderen Komponenten nicht in Einklang stehen ". ${ }^{16}$ Bei technischen Systemen, die aus unterschiedlichen, miteinander verbundenen Komponenten bestehen, hinkt mit einer gewissen Zwangsläufigkeit zu jedem Zeitpunkt eine dieser Komponenten hinterher. Wenn beispielsweise die Datenverarbeitungsgeschwindigkeit eines Computers von der Rechengeschwindigkeit des Prozessors abhängt, von der Zugriffsgeschwindigkeit des Speichermediums und von der Datenübertragungsgeschwindigkeit des sie verbindenden BusSystems, dann ist es zwangsläufig eine dieser drei Komponenten, die die Gesamtperformanz begrenzt und sich als natürliche Kandidatin für Innovationsbemühungen anbietet. Anschließend bildet dann eine der anderen beiden Komponenten den Engpass usw.

Diese Beobachtungen lassen sich dahingehend verallgemeinern, dass sich aus der Gestalt eines technischen Arrangements, d.h. aus dem ihm zu Grunde liegende Design und seiner Architektur Hinweise auf Richtungen für lohnenswerte Innovationen ergeben. Für die innovationsorientierende Wirksamkeit bestimmter technischer Designs prägen Nelson und Winter den Begriff des technologischen Regimes („technological regime“). Ihr Beispiel ist das Flugzeug Douglas DC3. Dessen Einführung in den 1930er Jahren, so die Autoren, „definierte ein bestimmtes technologisches Regime: Flugzeuge mit Metallhaut, Tiefflügel und Kolbenmotor. Die Ingenieure hatten ausgeprägte Vorstellungen über das Potenzial dieses Regimes. Mehr als zwei Jahrzehnte lang ging es bei der Innovation im Flugzeugbau im Wesentlichen darum, dieses Potenzial besser auszuschöpfen, die Triebwerke zu verbessern, die Flugzeuge zu vergrößern und sie effizienter zu machen. [...] F\&E-Strategien sind oft eng verbunden mit einem technologischen Regime [...]. Das Gefühl für das Potential, die Zwänge und die noch nicht genutzten Möglichkeiten, das in einem Regime implizit vorhanden ist, lenkt die Aufmerksamkeit der Ingenieure auf bestimmte Richtungen,

16 Hughes, The Evolution of Large Technological Systems, in: Bijker/Hughes/Pinch (Hrsg.), The Social Construction of Technological Systems. New Directions in the Sociology and History of Technology, 1987, S. 53. 
in denen Fortschritte möglich sind, und gibt starke Hinweise auf die Taktiken, die für die Erkundung dieser Richtung fruchtbar sein könnten." 17

Diese zumindest zeitweise Orientierung der innovierenden Akteure an einer bestimmten Architektur des betreffenden technischen Artefaktes oder Systems ist bei vielen technischen Innovationen von der Schreibmaschine über das Automobil bis hin zum Smartphone keine Heuristik nur eines einzelnen Unternehmens, sondern aller Unternehmen der betreffenden Branche bzw. des entsprechenden organisationalen Feldes. Mit Rebecca Henderson und Kim Clark kann man dann von einem dominanten Design ${ }^{18}$ sprechen: „Ein dominantes Design zeichnet sich durch eine Reihe zentraler Designkonzepte aus, die den Hauptfunktionen des Produkts entsprechen [...] und die in Komponenten verkörpert sind, und zusätzlich durch eine Produktarchitektur, die die Art und Weise der Integration dieser Komponenten bestimmt" ${ }^{19}$ Ein dominierendes Design enthält grundlegende Design-Entscheidungen, an denen sich die Ingenieure bis auf weiteres ganz selbstverständlich orientieren: „Nachdem das dominante Automobildesign akzeptiert worden war, haben die Ingenieure die Entscheidung für einen Benzinmotor nicht jedes Mal neu bewertet, wenn sie ein neues Design entwickelt haben. Sobald ein dominierendes Design festgelegt ist $[\ldots]$ nimmt der Fortschritt die Form von Verbesserungen der Komponenten im Rahmen einer stabilen Architektur an.“20

Die Heuristik der Orientierung an dominanten Designs und an den sie begleitenden technischen Regimen schafft Inseln relativer Kontinuität und relativer Handlungssicherheit in dem inhärent durch Diskontinuität und Unsicherheit geprägten Innovationsgeschehen. Diese Inseln können eher klein oder doch überschaubar sein, etwa wenn sich das dominante Design auf ein spezifisches technisches Artefakt bezieht, wie etwa im Fall des Nassrasierers, dessen Trajektorie die Entwickler inzwischen bis zum 6-KlingenRasierkopf geleitet hat. Oder sie können sich bildlich gesprochen zu veritablen Landmassen ausdehnen, wenn das dominante Design und das zugehörige Regime umfassendere technische Systeme definiert und dann, wie

17 Nelson/Winter, In Search of a Useful Theory of Innovation, S. 52f., eigene Übersetzung.

18 Der Begriff des dominanten Designs stammt ursprünglich von William Abernathy und James Utterback; vgl. dies., Patterns of Industrial Innovation, in: Technology Review 64 (1978), S. 44.

19 Henderson/Clark, Architectural Innovation: The Reconfiguration of Existing Product Technologies and the Failure of Established Firms, in: Administrative Science Quarterly 35(1) (1990), S. 14, eigene Übersetzung.

20 Henderson/Clark, Architectural Innovation, S. 14, eigene Übersetzung. 
etwa im Fall technischer Infrastruktursysteme, prägend für gesamtgesellschaftliche Strukturen und Prozesse wird.

Bei der Betrachtung technischer Systeme, die tief in gesellschaftliche Institutionen eingelassen sind, wird besonders deutlich, dass man es im Fall technologischer Regime tatsächlich mit sozio-technischen Regimen zu tun hat. In einem solchen sozio-technisch erweiterten Sinne definieren Arie Rip und René Kemp das technologische Regime als „Regelwerk oder Grammatik, das eingebettet ist in einen Komplex von Ingenieurpraktiken, Produktionsprozesstechnologien, Produkteigenschaften, Fertigkeiten und Verfahren, Arten und Weisen des Umgangs mit relevanten Artefakten und Personen, Arten und Weisen der Problemdefinition - die alle wiederum eingebettet sind in Institutionen und Infrastrukturen". ${ }^{21}$

Die Orientierung an sozio-technischen Regimen schafft aber nicht nur einfach Inseln relativer Kontinuität im Meer der mit Innovationen verbundenen Diskontinuitäten, diese Orientierung führt auch dazu, dass kontinuierliche und diskontinuierliche Prozesse im Innovationsgeschehen in komplexerer Weise miteinander verwoben werden als sich dies Schumpeter noch vorgestellt hatte. Drei dieser komplexeren Verwobenheiten möchte ich im Folgenden näher betrachten: (1) die Einbettung diskontinuierlicher Entwicklungen in kontinuierliche; (2) das Beharrungsvermögen soziotechnischer Regime und die Disruption aus der Nische; und (3) disruptivere Entwicklungen als Effekt des Beharrungsvermögens sozio-technischer Regime.

\section{Die Einbettung diskontinuierlicher Entwicklungen in kontinuierliche}

Ab den frühen 1980er Jahren etabliert sich die Unterscheidung zwischen inkrementellen und radikalen Innovationen, die auf den ersten Blick die Differenz zwischen dem Innovationsgeschehen innerhalb und außerhalb etablierter sozio-technischer Regime abzubilden scheint. Inkrementelle Innovationen sind danach Weiterentwicklungen, die das Potenzial eines eta-

21 Rip/Kemp, Technological Change, in: Rayner/Malone (Hrsg.), Human Choice and Climate Change, Vol. 2: Resources and Technology, 1998, S. 338; vgl. Kemp/Schot/ Hoogma, Regime Shifts to Sustainability through Processes of Niche Formation: The Approach of Strategic Niche Management, in: Technology Analysis \& Strategic Management 10(2) (1998), S. 175-198; Geels, Towards Sociotechnical Scenarios and Reflexive Anticipation: Using Patterns and Regularities in Technology Dynamics, in: Sørensen/Williams (Hrsg.), Shaping Technology, Guiding Policy: Concepts, Spaces and Tools, 2002, S. 359-381. 
blierten technischen Designs ausschöpfen, einer technologischen Trajektorie folgen und dabei nur kleinere Änderungen an bereits existierenden Produkten oder Prozessen darstellen. Als radikale Innovationen werden dagegen solche Neuerungen bezeichnet, die von der bestehenden Praxis abweichen, andere ingenieur- und naturwissenschaftlichen Grundlagen verwenden und dadurch oft neue Märkte eröffnen und ganz neue Anwendungen. ${ }^{22}$

Das Bild wird allerdings vielschichtiger, wenn man, wie es Henderson und Clark getan haben, genauer unterscheidet, worauf sich die grundlegenden Veränderungen radikaler Innovationen beziehen. In ihrem Artikel aus dem Jahre 1990 mit dem Titel Architectural Innovation ${ }^{23}$ unterscheiden Henderson und Clark zwischen zwei Formen grundlegender Neuerungen, die gemeinsam, aber auch unabhängig voneinander auftreten können: Dies ist zum einen die Entwicklung grundlegend neuer Komponenten. Die Entwicklung grundlegend neuer Komponenten im Rahmen unveränderter Produktarchitekturen bezeichnen Henderson und Clark als modulare Innovationen. Ein Beispiel ist die Ersetzung analoger Festnetztelefongeräte durch digitale.

Zum anderen kann die grundlegende Neuerung sich aber auch auf die Architektur beziehen und in der Entwicklung einer neuen Gesamtstruktur des technischen Systems bestehen, also einer neuen Art und Weise der Zusammensetzung und des Zusammenwirkens von Komponenten. Wenn dabei auf bereits bestehende Komponenten zurückgegriffen wird und deren Grundstruktur beibehalten wird, sprechen Henderson und Clark von architektonischen Innovationen. Ein anschauliches Beispiel für zwei technische Geräte mit unterschiedlichen Architekturen, aber im Grundsatz gleichen Komponenten, bilden der Deckenventilator und der Standventilator. $^{24}$

Für den Gesichtspunkt der Einbettung diskontinuierlicher Entwicklungen in die durch dominante Designs und sozio-technische Regime erzeugte Kontinuität sind die modularen Innovationen von besonderem Interes-

22 Vgl. Henderson/Clark, Architectural Innovation, S. 9; Freeman/Perez, Structural Crisis of Adjustment, Business Cycles and Investment Behaviour, in: Dosi et al. (Hrsg.), Technical Change and Economic Theory, 1988, S. 45f.; Dewar/Dutton, The Adoption of Radical and Incremental Innovations: An Empirical Analysis, in: Management Science 32(11) (1986), S. 1422f.

23 Vgl. Henderson/Clark, The Reconfiguration of Existing Product Technologies and the Failure of Established Firms, in: Administrative Science Quarterly 35(1) (1990), S. 9-30.

24 Vgl. Henderson/Clark, Architectural Innovation, S. $12 \mathrm{f}$. 
se. Einer in der Innovationsforschung weit verbreiteten Sichtweise zufolge konzentrieren sich die etablierten Unternehmen einer Branche auf inkrementelle Innovationen im Rahmen etablierter sozio-technischer Regime. D.h. sie wenden die Heuristik an, im Bereich ihrer bestehenden Fähigkeiten und Ausstattungen nach vielversprechenden Neuerungen zu suchen. Umgekehrt haben etablierte Unternehmen dieser verbreiteten Sichtweise zufolge große Schwierigkeiten mit radikalen Innovationen. Der von Henderson und Clark angebotene differenziertere Blick auf radikale Innovationen zeigt dagegen, dass diese besondere Schwierigkeit etablierter Unternehmen sich vor allem auf radikalen Innovationen richtet, die architektonische Innovationen beinhalten, weniger dagegen aber auf grundlegende Neuerungen im Bereich modularer Innovationen.

Wenn es um das Ausschöpfen von Innovationspotenzialen im Rahmen etablierter sozio-technischer Regime geht, dann haben etablierte Unternehmen nicht nur bei inkrementellen Innovationen die Nase vorn, sondern auch bei denjenigen radikalen Innovationen, die sich innerhalb dieses Referenzrahmens als vielversprechend erweisen. Und dies sind typischerweise modulare Innovationen, die sich im Rahmen der Architektur eines dominanten Designs auf neue Komponenten richten - auf neue Komponenten, die bisherige Komponenten ersetzen sollen, deren Entwicklungspotenzial ausgeschöpft ist. Dieses Innovationsmuster zeigt Clayton Christensen am Beispiel der Entwicklung der Speicherplatten und der Lese- und Schreibköpfe von Computerlaufwerken.

Er schreibt: „In den 1970er Jahren waren sich einige Hersteller durchaus darüber bewusst, dass die Speicherkapazität oxydbeschichteter Platten an ihre Grenzen stößt. Um dennoch die gewohnten Leistungsverbesserungen zu erzielen, begannen sie, sich mit anderen Technologien zu beschäftigen. Sie experimentierten etwa mit Dünnfilmbeschichtungen, in der Hoffnung, auf diese Weise die kundenseitig bereits gewohnte Kapazitätssteigerung zu erreichen. [...] Expertenschätzungen zufolge nahm die Entwicklung mehr als 8 Jahre in Anspruch und sollte die Pioniere der Dünnfilm-Platten-Technologie - IBM, Control Data, Digital Equipment, Storage Technology und Ampex - jeweils mehr als 50 Millionen Dollar kosten. Zwischen 1984 und 1986 führten zwei Drittel der Marktteilnehmer Laufwerke mit Dünnfilmplatten ein. Die überwältigende Mehrheit der Anbieter waren etablierte Unternehmen. [...] Auch bei der Einführung von Dünnfilmköpfen stoßen wir auf das gleiche Muster. Die Hersteller der Ferrit-Köpfe sahen bereits 1965 deutliche Grenzen dieser Technologie erreicht. Schließlich glaubten 1981 die meisten, dass die Präzision kaum weiter gesteigert werden kann. In der Forschung und Entwicklung wendete man sich nun der Dünnfilm-Technologie zu. [...] Wie bereits bei den Dünnfilm-Speicher- 
platten, so zeigt sich auch bei den Dünnfilmköpfen ein technologischer Wandel, den nur etablierte Unternehmen meistern. IBM und seine Konkurrenten investierten jeweils 100 Millionen Dollar und mehr für die Entwicklung der Dünnfilmköpfe. Dieses Muster wiederholte sich schließlich auch bei der MR-Technologie [d.h. der magnetoresistiven Speicherung, Anm. ISS]: Die branchengrößten Unternehmen - IBM, Seagate und Quantum - führten das Rennen unbestritten an."25

Das Innovationsmuster, das in diesen Beispielen zum Ausdruck kommt, ist deshalb so interessant, weil es zeigt, dass dominante Designs und etablierte sozio-technische Regimen heuristische Orientierungen hervorbringen, die von den innovierenden Akteuren als so verlässlich betrachtet werden, dass sie die Grundlage für hoch riskante und ausgesprochen kostenträchtige radikale Innovationen bilden können.

\section{Das Beharrungsvermögen sozio-technischer Regime und die Disruption aus der Nische}

Die heuristische Orientierung an sozio-technischen Regimen hat zudem Auswirkungen auf das grundsätzliche Kräfteverhältnis zwischen kontinuierlichen und diskontinuierlichen Neuerungen. Schumpeter war noch davon ausgegangen, dass diskontinuierliche Entwicklungen gleichsam automatisch den Motor des ökonomischen Wandels bilden, weil sie einen sich beständig wiederholenden Prozess in Gang setzen, in dem „sich die neuen Kombinationen durch das Niederkonkurrieren der alten durchsetzen“. ${ }^{26}$ Die Orientierung an sozio-technischen Regimen verleiht einmal etablierten dominanten Designs dagegen ein beträchtliches Beharrungsvermögen und dies auch gegenüber den möglicherweise verheißungsvolleren, diese Bahn verlassenden und in diesem Sinne disruptiven Alternativen.

$\mathrm{Zu}$ diesem Beharrungsvermögen trägt die Vielzahl von inkrementellen Innovationen bei, die die Orientierung an etablierten sozio-technischen Regimen mit sich bringt. Ein großer Teil aller technologischen Innovationen, so konstatieren Stephen Kline und Nathan Rosenberg bereits 1986, „erfolgt in Form von sehr kleinen Änderungen, wie z.B. geringfügigen Änderungen im Design einer Maschine, die sie besser für bestimmte sehr spezifische Endanwendungen eignet, oder die ihre Herstellung einfacher und

25 Christensen, The Innovator's Dilemma: Warum etablierte Unternehmen den Wettbewerb um bahnbrechende Innovationen verlieren, 2011 [1997], S. 29.

26 Schumpeter, Theorie der wirtschaftlichen Entwicklung, S. 101. 
damit kostengünstiger machen [...] Die meisten wichtigen Innovationen durchlaufen in ihrem Leben drastische Veränderungen - Veränderungen, die ihre wirtschaftliche Bedeutung völlig verändern können und oft auch tun. Die späteren Verbesserungen einer Erfindung nach ihrer ersten Einführung können wirtschaftlich wesentlich wichtiger sein als die anfängliche Verfügbarkeit der Erfindung in ihrer ursprünglichen Form. “27 Umgekehrt bedeutet dies, dass disruptive Innovationen außerhalb des Rahmens eines etablierten sozio-technischen Regimes anfangs stets erst die grobschlächtige Rohform der Technologie sind, zu der sie nachfolgend durch zunehmendes Ausschöpfen ihres Potenzials werden können. Joel Mokyr bezeichnet solche grundlegenden Innovationen in ihrer Anfangsphase deshalb auch als „hopeful monstrosities“, als hoffnungsverheißende Monstrositäten. ${ }^{28}$ Unter den Bedingungen der Konkurrenzwirtschaft ist es in dieser Konstellation dann eher wahrscheinlich, dass sich die alten Kombinationen durch das Niederkonkurrieren der neuen durchsetzen.

Um konkurrenzfähig zu den Technologien eines etablierten sozio-technischen Regimes werden zu können, benötigen die hoffnungsverheißenden Monstrositäten deshalb Schutzräume, in denen sie reifen können, in denen also genau jene inkrementellen Lern- und Verbesserungsprozesse ebenfalls stattfinden, die die Heuristik der Orientierung an einem dominanten Design mit sich bringt. Die Bedeutung und Wirkungsweise solcher Schutzräume für grundlegende Innovationen diskutiert die Innovationsforschung seit gut zwei Jahrzehnten unter dem Begriff der Nische. ${ }^{29}$ Bei diesen Nischen kann es sich um Marktnischen handeln, also um Sondermärkte mit spezifischen Gegebenheiten, in denen die Vorteile selbst einer noch grobschlächtigen Neuerung so groß sind, dass sie die Nachteile ihrer Unausgereiftheit überwiegen. Beispielsweise hat sich das Dampfschiff in der Nische der Flussschifffahrt entwickelt, als es auf offenem Wasser noch keine Konkurrenz für das Segelschiff war. Auf dem Fluss bot die technisch noch anfällige motorisierte Fortbewegung den dort unschlagbaren Vorteil der Fortbewegung im direkten Gegenwind. Nischen können aber auch durch technologiepolitische Maßnahmen erzeugt werden. So hat beispiels-

27 Kline/Rosenberg, An Overview of Innovation, in: Landau/Rosenberg (Hrsg.), The Positive Sum Strategy. Harnessing Technology for Economic Growth, 1986, S. $282 \mathrm{f}$.

28 Mokyr, Evolutionary Biology, Technological Change and Economic History, in: Bulletin of Economic Research, 43(2) (1991), S. 142.

$29 \mathrm{Kemp} / \mathrm{Schot} / \mathrm{Hoogma}$, Regime Shifts to Sustainability through Processes of Niche Formation, S. 183f.; Geels/Schot, Typology of Sociotechnical Transition Pathways, in: Research Policy 36(3) (2007), S. 400. 
weise das Stromeinspeisungsgesetz (und sein Nachfolger, das ErneuerbareEnergien-Gesetz), eine Nische für Techniken der Stromerzeugung aus erneuerbaren Energien geschaffen.

$\mathrm{Ob}$ das Potenzial, das in einer disruptiven Innovation schlummert, gesellschaftlich zum Tragen kommt oder ungenutzt bleibt, hängt unter diesen Gegebenheiten wesentlich davon ab, ob gerade eine passende Marktnische vorhanden ist oder geschaffen werden kann, oder ob es interessierten Akteuren aus Wirtschaft, Wissenschaft, Politik oder gesellschaftlicher Öffentlichkeit in anderer Weise gelingt, die gesellschaftliche Umwelt für die betreffende Neuerung und deren Nutzung so zu verändern, dass eine entsprechende Nische entsteht.

Damit aber wird das Geschäft des Innovierens in noch einem anderen Sinne ein Handlungsbereich, der sich der Logik des Ökonomischen entzieht, als Schumpeter es sich noch vorgestellt hatte. Schumpeter hatte zwar bereits gesehen, dass ökonomische Kalküle des Berechnens von Preisen und Mengen nicht auf Innovationsentscheidungen anwendbar sind. Aber er war davon ausgegangen, dass in der wiederholten Durchsetzung diskontinuierlicher Entwicklungen dann doch die Logik ökonomischer Konkurrenz zum Ausdruck kommt und den Anreiz für entsprechendes innovatorisches Handeln bereitstellt. Prozesse der Herstellung und Stabilisierung von Nischen folgen dagegen sehr viel stärker der Logik des Politischen, und zwar nicht nur dann, wenn politische Regulierungen die Nische erzeugen. Denn Nischen zu erzeugen bedeutet, Verbündete zu finden und Koalitionen von Akteuren zu bilden, die an der gesellschaftlichen Durchsetzung der Neuerung interessiert sind. Die Interessen an der Durchsetzung müssen dabei keineswegs allein ökonomischer Art sein, sondern sie können das ganze Spektrum politischer, normativer und weltanschaulicher Einstellungen umfassen.

\section{Disruptivere Entwicklungen als Effekt des Beharrungsvermögens sozio- technischer Regime}

Die Kehrseite der Steigerung der Kontinuität des Innovationshandelns durch die Orientierung an etablierten sozio-technischen Regimes ist, dass die Brüche, die mit diskontinuierlichen Entwicklungen einhergehen, umso tiefer werden können. Dies ist die letzte der drei Verwobenheiten kontinuierlicher und diskontinuierlicher Prozesse im Innovationsgeschehen, 
die ich hervorheben möchte. Sie ist besonders prägnant von Christensen ${ }^{30}$ herausgearbeitet worden, dessen Arbeiten es dann auch ganz wesentlich waren, die den Begriff der Disruption in das Vokabular der Innovationsforschung gebracht haben.

Christensen geht es um die Erklärung des Phänomens, dass die etablierten Unternehmen einer Branche es häufig versäumen, rechtzeitig auf jene Innovationen zu setzen, die die Spielregeln in dieser Branche grundlegend ändern. So hat beispielsweise der Kataloghandel die Entwicklung des Online-Handels verschlafen, die großen Plattenlabel waren viel zu lange desinteressiert am Geschäft mit dem Verkauf von MP3-Musikdateien und die großen Fluggesellschaften waren Nachzügler im Geschäft mit Billigflügen. Dieses Verhalten ist deshalb besonders erklärungsbedürftig, weil es vielfach zur Folge hatte, dass diese führenden Unternehmen große Marktanteile verloren oder ganz vom Markt verschwanden.

Christensens Erklärung besteht im Kern aus zwei Teilen: Erstens: Die Innovationen, die später zu einer Bedrohung der Geschäftsmodelle der etablierten Firmen einer Branche werden, entwickeln sich in Marktnischen. Zweitens: Aus der Perspektive der Geschäftsmodelle der etablierten Unternehmen der betreffenden Branche sind diese Nischenmärkte ganz uninteressant. Deshalb kümmern sie sich nicht um das Innovationsgeschehen in dieser Nische, und wenn sie die Bedrohung erkennen, sind sie gegenüber den Unternehmen in dieser Nische bereits deutlich ins Hintertreffen geraten.

Der erste Teil des Argumentes knüpft an die schon angesprochenen Überlegungen zur Reifung bahnverändernder Innovationen in Marktnischen an. Es ist zunächst eine spezifische Gegebenheit der Nische, die eine Innovation trotz ihrer Unausgereiftheit dort zum Einsatz bringt - im Fall des Flussdampfschiffs ist dies der Umstand, dass Flüsse meist zu schmal sind, um mit einem Segelschiff gegen den Wind zu kreuzen. Mit zunehmender Reifung der Innovation in der Nische - durch die angesprochene Vielzahl inkrementeller Innovationen an dem gegebenen Design - kann es dann aber geschehen, dass sie ihre Nachteile gegenüber den bereits ausgereiften Problemlösungen des etablierten sozio-technischen Regimes verliert. Wenn die Nachteile verschwunden sind, können die zunächst nur nischenspezifisch wirksamen Vorteile dieser Innovation nun auch für den Hauptmarkt attraktiv werden, was die Innovation gegenüber der bisherigen Problemlösung zur attraktiveren Alternative werden lassen kann.

30 Christensen, The Innovator's Dilemma: Warum etablierte Unternehmen den Wettbewerb um bahnbrechende Innovationen verlieren, 2011 [1997]. 
Für den zweiten Teil des Argumentes sind die Heuristiken der Orientierung an sozio-technischen Regimen von wesentlicher Bedeutung. Solange die Unternehmen einer Branche sich an einem etablierten sozio-technischen Regime orientieren, konkurrieren sie miteinander mit Innovationen, die darauf zielen, das Potenzial des dominanten Designs weiter auszuschöpfen. Sie konzentrieren sich, so Christensen, auf Innovationen, die „darauf gerichtet sind, die Leistungsfähigkeit von vorhandenen Produkten entlang der zentralen Kundenanforderungen in bestehenden Märkten zu steigern." ${ }^{1}$ Die dahinterstehende Rationalität ist, dass eine kleine Verbesserung des Marktanteils in dem großen Hauptmarkt ökonomisch mehr wert ist als die Vorherrschaft in einem kleinen Nischenmarkt. Das resultierende ökonomische Desinteresse an den Nischenmärkten führt dann dazu, dass Bedrohungen durch Disruption aus der Nische zu spät erkannt werden.

Natürlich sind den Akteuren im Innovationsgeschehen, die dieser Rationalität folgen, deren unerwünschte Nebenfolgen inzwischen bekannt, und die etablierten Unternehmen einer Branche haben eine Reihe von Strategien entwickelt, um mit solchen Bedrohungen aus der Nische umzugehen. Hier soll abschließend nur eine, besonders radikale dieser Strategien angesprochen werden, nämlich die Strategie der großen Internetkonzerne und der durch Risikokapital finanzierten Startups des Silicon Valley, von ihnen kontrollierte neue Märkte zu schaffen, und sie eine Zeitlang als Nischen zu subventionieren, aber mit dem Ziel, daraus in kurzer Zeit den neuen Hauptmarkt zu machen. Bekannte Beispiele für diese Strategie sind iTunes, Uber, Airbnb oder Netflix.

Neu daran ist nicht, dass die Akteure disruptiver Innovationen einen wesentlichen Teil ihrer Arbeit darauf richten, die politische, rechtliche, ökonomische und infrastrukturelle Umgebung ihrer Neuerung so zu beeinflussen, dass diese Innovation gegenüber dem etablierten sozio-technischen Regime eine Chance bekommt. So war bereits Thomas Edison vorgegangen. Neu ist allerdings die durch Internetplattformen technisch ermöglichte Strategie, diese Nische von Anfang an als den neuen Hauptmarkt anzulegen. Allgemein gilt: Je mehr Arbeit in die Weiterentwicklung eines dominanten Designs geflossen ist und je mehr Zeit ein sozio-technisches Regime hatte, sich zu etablieren, desto tiefer ist der Bruch hin zu einem neuen dominanten Design. Was jetzt aber noch hinzukommt, ist: Wenn dieser Bruch dann nicht mehr durch die Zeit des Heranreifens der neuen Problemlösung in der Nische abgefedert wird, wird der technische und soziale Wandel umso disruptiver.

31 Christensen, ebd., S. 6. 